\title{
FORCED NONLINEAR VIBRATION OF SHALLOW SHELL AND APPLICATION TO TECHNICAL DIAGNOSIS
}

\author{
Le Ngoc Thach, Tran Van Lien \\ Hanoi University of Civil Engineering
}

\begin{abstract}
This paper presents direct and inverse problems of forced nonlinear vibration of shallow shell. From that the method of establishment and solution of technical diagnosis for shallow shell under the vibrated load is proposed. The diagnosis criterion is the minimum of the difference between the square of experimental and theoretical data. The diagnosed parameters are the external loads and the reactions arose at the boundaries.
\end{abstract}

\section{INTRODUCTION}

We have many different approaches to solve a technical diagnosis problem. One of those is employment of experiment determined and theoretical computed frequencies of the forced vibration of structure in order to determine the unknown technical parameters.

When solving a technical diagnosis problem of structures, the numerical results of a program on structural analysis of the direct problem are often employed. However, to the date, these numerical results are mostly given for linear problems with small strain assumption. Even, the linear problems have many advantages, but do not completely model the behavior the structures, especially in the case of dynamic problems.

In this research direction, in [7] the technical diagnosis problem and its solution for shallow shell are presented, but just for the case of free vibration. In this paper, based on the direct and inverse problems of forced vibration the authors present the method to establish and solve the technical diagnosis problem for shallow shell under the vibrated loads.

The diagnosis criterion is the minimum of the difference between the square of experimental and theoretical data. The diagnosed parameters are the external loads and the reactions arose at the boundaries.

\section{MODEL CONSTRUCTION}

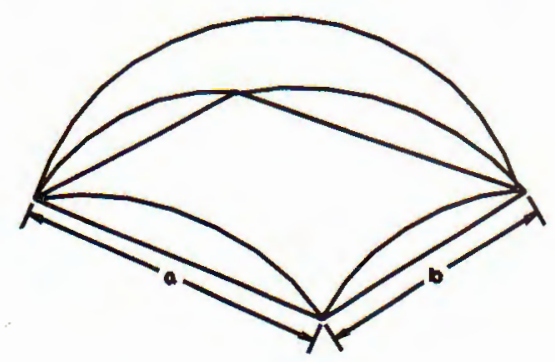

Fig. 1. Shallow shell with rectangular base 
Considering the forced vibration of shallow shell (cylindrical, spherical) with rectangular base, the sizes of $a \times b \times h$ and with four simply supported edges are shown in Fig. 1. In order to examine the proposed problem, we adopte herewith the nonlinear forced vibrating equation of shallow shell as follows [3]:

$$
\left\{\begin{array}{l}
\frac{D}{h} \nabla^{4} W=L(W, \phi)+\nabla_{k}^{2} \phi+\frac{q}{h}-\frac{\gamma}{g} \frac{\partial^{2} W}{\partial t^{2}}, \\
\frac{1}{E} \nabla^{4} \phi=-\frac{1}{2} L(W, W)-\nabla_{k}^{2} W,
\end{array}\right.
$$

in which

$$
\begin{aligned}
& L(W, \phi)=\frac{\partial^{2} W}{\partial x^{2}} \frac{\partial^{2} \phi}{\partial y^{2}}+\frac{\partial^{2} W}{\partial y^{2}} \frac{\partial^{2} \phi}{\partial x^{2}}-2 \frac{\partial^{2} W}{\partial x \partial y} \frac{\partial^{2} \phi}{\partial x \partial y} ; \\
& \nabla_{k}^{2}=\frac{1}{R_{y}} \frac{\partial^{2}}{\partial x^{2}}+\frac{1}{R_{x}} \frac{\partial^{2}}{\partial y^{2}} ; \quad \nabla^{4}=\frac{\partial^{4}}{\partial x^{4}}+2 \frac{\partial^{4}}{\partial x^{2} \partial y^{2}}+\frac{\partial^{4}}{\partial y^{4}}
\end{aligned}
$$

and $D=\frac{E h^{3}}{12\left(1-\mu^{2}\right)}$ is the cylindrical stiffness, $\mu$ is the Poisson ratio, $E$ is the elastic modulus, $W$ is the bending function, $\phi$ is the stress function, $\gamma$ is the weight density, $g$ is the gravity acceleration, $\nabla^{4}$ is the double Laplace operator. The curvatures of the cylindrical shell to $x$ and $y$ coordinates are respectively $R_{x}=\infty, R_{y}=R=$ const. The curvatures of the spherical shell to $x$ and $y$ coordinates are respectively $R_{x}=R_{y}=R=$ conts. The curvatures of the plate are $R_{x}=R_{y}=\infty . q(x, y, t)$ is the lateral load of the shell or plate.

Choose the deflection function as double triangle function

$$
W(x, y, t)=f(t) \sin \left(\frac{n \pi x}{a}\right) \sin \left(\frac{m \pi y}{b}\right) .
$$

This solution always satisfies the boundaries condition with four simply supported edges in the vibration directions as following

$$
\begin{gathered}
\left.W\right|_{x=0}=\left.W\right|_{x=a}=\left.W\right|_{y=0}=\left.W\right|_{y=b}=0 \\
\left.\frac{\partial^{2} W}{\partial x^{2}}\right|_{x=0}=\left.\frac{\partial^{2} W}{\partial x^{2}}\right|_{x=a}=\left.\frac{\partial^{2} W}{\partial y^{2}}\right|_{y=0}=\left.\frac{\partial^{2} W}{\partial y^{2}}\right|_{y=b}=0 .
\end{gathered}
$$

Substituting (2.3) into the second equation of (2.1), we obtain equation of the stress function $\phi$

$$
\frac{1}{E} \nabla^{4} \phi=\frac{1}{2} f^{2}(t) \frac{n^{2} m^{2} \pi^{4}}{a^{2} b^{2}}\left(\cos \frac{2 n \pi x}{a}+\cos \frac{2 m \pi y}{b}\right)+f(t)\left(\frac{n^{2} \pi^{2}}{R_{y} a^{2}}+\frac{m^{2} \pi^{2}}{R_{x} b^{2}}\right) \sin \frac{n \pi x}{a} \sin \frac{m \pi x}{b} .
$$

The solution of the homogenous differentiation equation (2.4) contains the homogenous solution having polynomial form and a trigonometry form particular solution. Supposing the boundary loads $P_{x}$ and $P_{y}$ are constant, the $\phi$ function can be chosen in the form

$$
\begin{aligned}
\phi= & P_{x} \frac{y^{2}}{2}+P_{y} \frac{x^{2}}{2}+\frac{E f^{2}(t)}{32}\left(\frac{m^{2} a^{2}}{n^{2} b^{2}} \cos \frac{2 n \pi x}{a}+\frac{n^{2} b^{2}}{m^{2} a^{2}} \cos \frac{2 m \pi y}{b}\right)+ \\
& +\frac{E f(t)}{\pi^{2}\left(m^{2} a^{2}+n^{2} b^{2}\right)^{2}}\left(\frac{m^{2} a^{4} b^{2}}{R_{y}}+\frac{n^{2} a^{2} b^{4}}{R_{y}}\right) \sin \frac{n \pi x}{a} \sin \frac{m \pi y}{b} .
\end{aligned}
$$


From (2.5) we can calculate the stress components as

$$
\begin{aligned}
\sigma_{x}=\frac{\partial^{2} \phi}{\partial y^{2}}= & -\frac{E f^{2} n^{2} \pi^{2}}{8 a^{2}} \cos \left(\frac{2 m \pi y}{b}\right)-\frac{E f n^{2} m^{2} a^{2} b^{2}}{R_{y}\left(m^{2} a^{2}+n^{2} b^{2}\right)^{2}} \sin \left(\frac{n \pi x}{a}\right) \sin \left(\frac{m \pi y}{b}\right) \\
& -\frac{E f m^{4} a^{4}}{R_{x}\left(m^{2} a^{2}+n^{2} b^{2}\right)^{2}} \sin \left(\frac{n \pi x}{a}\right) \sin \left(\frac{m \pi y}{b}\right)+P_{x} \\
\tau_{x y}=- & \frac{\partial^{2} \phi}{\partial x \partial y}=-E f\left[\frac{m n^{3} a b^{3}}{R_{y}\left(m^{2} a^{2}+n^{2} b^{2}\right)^{2}}+\frac{m^{3} n a^{3} b}{R_{x}\left(m^{2} a^{2}+n^{2} b^{2}\right)^{2}}\right] \cos \left(\frac{n \pi x}{a}\right) \cos \left(\frac{m \pi y}{b}\right) \\
\sigma_{y}=\frac{\partial^{2} \phi}{\partial x^{2}}= & -\frac{E f^{2} m^{2} \pi^{2}}{8 b^{2}} \cos \left(\frac{2 n \pi x}{a}\right)-\frac{E f n^{4} b^{4}}{R_{y}\left(m^{2} a^{2}+n^{2} b^{2}\right)^{2}} \sin \left(\frac{n \pi x}{a}\right) \sin \left(\frac{m \pi y}{b}\right) \\
& -\frac{E f m^{2} n^{2} a^{2} b^{2}}{R_{x}\left(m^{2} a^{2}+n^{2} b^{2}\right)^{2}} \sin \left(\frac{n \pi x}{a}\right) \sin \left(\frac{m \pi y}{b}\right)+P_{y} .
\end{aligned}
$$

Choose the load function in the form

$$
q=q(x, y, t)=f_{0} \sin \frac{n \pi x}{a} \sin \frac{m \pi y}{b} \cos (\omega t) .
$$

In order to get the vibration spectrum of the structure we need to determine the equation of the amplitude function $f(t)$. We adopted herewith the Bubnov - Galerkin method for the first equation of (2.1), namely

$$
\int_{0}^{a} \int_{0}^{b}\left[\frac{D}{h} \nabla^{4} W-L(W, \phi)-\nabla_{k}^{2} \phi-\frac{q}{h}+\frac{\gamma}{g} \frac{\partial W^{2}}{\partial t^{2}}\right] \sin \frac{n \pi x}{a} \sin \frac{m \pi y}{b} d x d y=0 .
$$

Performing the integral in (2.8) we get the differentiation equation under the form

$$
F(t)=0 \text {. }
$$

The equation (2.9) describes the nonlinear vibration of the function $f(t)$ for vibration modes of the system.

\subsection{Rectangular plates}

For the problem of rectangular plates $\left(R_{x}=R_{y}=\infty\right)$, from (2.9) we obtain the nonlinear vibration equation for the system under the form

$$
\begin{aligned}
\frac{\gamma}{g} \frac{d^{2} f}{d t^{2}}+ & {\left[D \frac{\pi^{4}}{h}\left(\frac{m^{2}}{b^{2}}+\frac{n^{2}}{a^{2}}\right)^{2}+P_{x}\left(\frac{n \pi}{a}\right)^{2}+P_{y}\left(\frac{m \pi}{b}\right)^{2}\right] f(t)+} \\
& +\frac{E \pi^{4}}{16}\left(\frac{m^{4}}{b^{4}}+\frac{n^{4}}{a^{4}}\right) f^{3}(t)=\frac{f_{0} \cos (\omega t)}{h}
\end{aligned}
$$

or

in which

$$
\ddot{f}+\omega_{0}^{2} f+\alpha f^{3}=F \cos \omega t
$$

$$
\begin{aligned}
& \omega_{0}^{2}=\frac{g}{\gamma}\left[D \frac{\pi^{4}}{h}\left(\frac{m^{2}}{b^{2}}+\frac{n^{2}}{a^{2}}\right)^{2}+P_{x}\left(\frac{n \pi}{a}\right)^{2}+P_{y}\left(\frac{m \pi}{b}\right)^{2}\right] \\
& \alpha=\frac{E \pi^{4}}{16} \frac{g}{\gamma}\left(\frac{m^{4}}{b^{4}}+\frac{n^{4}}{a^{4}}\right) ; \quad F=\frac{g f_{0}}{\gamma h} .
\end{aligned}
$$


In order to obtain the solution of equation (2.11) we apply herewith the iterative method. For the first approximation we assume

$$
f_{1}(t)=A \cos (\omega t)
$$

in which $A$ is the unknown constant. Substituting (2.13) into (2.12), we get differentiation equation for the second approximation

$$
\ddot{f}_{2}=-A \omega_{0}^{2} \cos (\omega t)-A^{3} \alpha \cos ^{3}(\omega t)+F \cos (\omega t)
$$

or

$$
\ddot{f}_{2}=-\left(A \omega_{0}^{2}+\frac{3}{4} A^{3} \alpha-F\right) \cos (\omega t)-\frac{1}{4} A^{3} \alpha \cos (3 \omega t) .
$$

Integrating equation (2.15) with the initial condition equals to 0 , we get the second approximation solution which is cyclic vibration

$$
f_{2}(t)=\frac{1}{\omega^{2}}\left(A \omega_{0}^{2}+\frac{3}{4} A^{3} \alpha-F\right) \cos (\omega t)+\frac{A^{3} \alpha}{36 \omega^{2}} \cos (3 \omega t) .
$$

Since the solution $f_{1}(t)$ from equation (2.13) and $f_{2}(t)$ from equation (2.16) are approximations of $(2.11)$, so that we have

$$
A=\frac{1}{\omega^{2}}\left(A \omega_{0}^{2}+\frac{3}{4} A^{3} \alpha-F\right),
$$

so

$$
\omega^{2}=\omega_{0}^{2}+\frac{3}{4} A^{2} \alpha-\frac{F}{A},
$$

or

$$
\begin{aligned}
\omega^{2}= & \frac{g E}{\gamma}\left[\frac{\pi^{4} h^{2}}{12\left(1-\mu^{2}\right)}\left(\frac{n^{2}}{a^{2}}+\frac{m^{2}}{b^{2}}\right)^{2}\right]+\frac{\pi^{2} g}{\gamma}\left(\frac{P_{x} n^{2}}{a^{2}}+\frac{P_{y} m^{2}}{b^{2}}\right)+ \\
& +\frac{3}{64} \frac{\pi^{4} g E}{\gamma}\left(\frac{n^{4}}{a^{4}}++\frac{m^{4}}{b^{4}}\right) A^{2}-\frac{g f_{0}}{\gamma A h} .
\end{aligned}
$$

When $\alpha=0$, from (2.18) we obtain the well known formula of the linear vibration theory (Fig. 2.a)

$$
A=\frac{F}{\omega^{2}-\omega_{0}^{2}}
$$

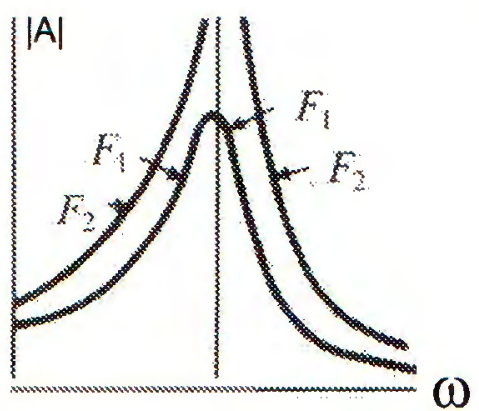

a)

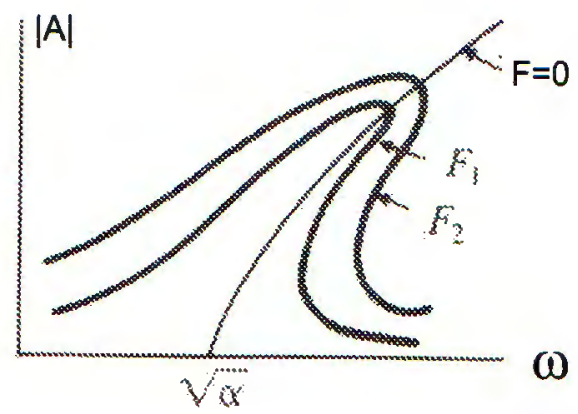

b)

Fig. 2. Respone curves of equation (2.11) 
For the case of nonlinear vibration $(\alpha \neq 0)$, the frequency $\omega$ is a function of the vibration amplitude $A$, nonlinear coefficient $\alpha$ and the amplitude of the external loads $F$ (Fig. 2.b).

\subsection{Shallow shell}

In order to determine the frequency $\omega$ of the system, we can write the system vibration under the form

$$
f(t)=A \cos \omega t
$$

From the differentiation equation (2.9), using the Bubnov - Galerkin method

$$
\int_{0}^{2 \pi} \omega F(t) \cos (\omega t) d t=0 .
$$

Substituting $f(t)$ from (2.21) in to (2.22), after performing the integral over a period of vibration, we get the formula expressing the relationship between the frequency $\omega$ and amplitude of the forced vibration $A$ of the cylindrical, shallow shells as:

$$
\begin{aligned}
\omega_{m, n}^{2}= & \frac{g E}{\gamma}\left[\frac{\pi^{4} h^{2}}{12\left(1-\mu^{2}\right)}\left(\frac{n^{2}}{a^{2}}+\frac{m^{2}}{b^{2}}\right)^{2}+\frac{\left(\frac{m^{2}}{b^{2} R_{x}}+\frac{n^{2}}{a^{2} R_{y}}\right)^{2}}{\left(\frac{n^{2}}{a^{2}}+\frac{m^{2}}{b^{2}}\right)^{2}}\right]+ \\
& +\frac{\pi^{2} g}{\gamma}\left(\frac{P_{x} n^{2}}{a^{2}}+\frac{P_{y} m^{2}}{b^{2}}\right)+\frac{3}{64} \frac{\pi^{4} g E}{\gamma}\left(\frac{n^{4}}{a^{4}}+\frac{m^{4}}{b^{4}}\right) A^{2}-\frac{g f_{0}}{\gamma A h} .
\end{aligned}
$$

The obtained expression (2.23) is an extension of (2.19). To this point, we can understand the meaning of each term on the right hand side of (2.19) or (2.23):

- The first term is the natural frequency of the linear problem of the rectangular plates with four simply supported edges.

- The summation of the first two terms is solution of the considering problem for the case of small deformation and strain.

- The third term is corresponding to the effect of axial force uniformly distributed on the boundaries.

- The fourth term is expressing the effect of nonlinear part to the natural frequency of the system.

- The last term describes the effect of the ratio between amplitude of the external force and amplitude of the forced vibration.

\section{ESTABLISHMENT OF INVERSE - TECHNICAL DIAGNOSIS PROBLEM}

Based on the result of the above proposed problem on vibration of plates and shell (expression (2.23)), the inverse - technical diagnosis problem to assess the behavior of the shell when knowing the natural frequencies is established this section.

Considering a cylindrical or spherical shell with rectangular base under compressive loads $P_{x}, P_{y}$ and $q(x, t)$ having the form (2.7) on the boundaries. Assuming that some natural frequencies of the shell have been measured, our problem is that how to determine 
the unknown external loads $P_{x}, P_{y}$ and the amplitude of the external load from the known natural frequencies $\omega_{1}^{*}, \omega_{2}^{*}, \ldots, \omega_{m}^{*}$ of the shell.

The minimum of difference between the square of the measured frequencies $\vec{\omega}^{*}=$ $\left\{\omega_{1}^{*}, \omega_{2}^{*}, . ., \omega_{m}^{*}\right\}$ and computed frequencies $\vec{\omega}=\left\{\omega_{1}, \omega_{2}, . ., \omega_{m}\right\}$ according to $(2.23)$ is chosen as the criterion for evaluating the diagnosis parameters.

$$
F\left(f_{0}, P_{x}, P_{y}\right)=\left\|\vec{\omega}-\vec{\omega}^{*}\right\|=\sum_{i=1}^{m}\left|\omega_{i}-\omega_{i}^{*}\right|^{2} \rightarrow \min .
$$

The technical diagnosis problem of the unknown parameters $\left\{f_{0}, P_{X}, P_{Y}\right\}$ of the shell deduces to the problem of minimizing of the difference function (3.1) with the constrains

$$
\left\{\begin{array}{c}
f\left(f_{0}, P_{x}, P_{y}\right) \rightarrow \min \\
f_{0} \geq 0 ; \quad P_{x} \leq 0 ; \quad P_{y} \leq 0
\end{array}\right.
$$

To solve this problem, we can employ the function LSQLIN in Optimization Toolbox of MatLab [1] with command

$[x$, resnorm, residual, exitflag, output, lambda $]=$ in which

Isqlin(C,Tsodo, [ ]. [ ], [ ], [ ], Ib, ub, x0, options, varargin)

- Tsodo is vector of measured frequencies.

- $C$ is vector of coefficient of parameters $f_{0}, P_{x}, P_{y}$ in (2.23).

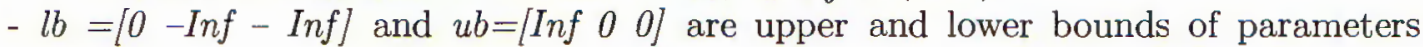
$f_{0}, P_{x}, P_{y}$.

The input parameters are the measured frequencies of the shell $\vec{\omega}^{*}=\left\{\omega_{1}^{*}, \omega_{2}^{*}, \ldots, \omega_{m}^{*}\right\}$, and the results are the diagnosis parameters $\left\{f_{0}, P_{x}, P_{y}\right\}$

\section{NUMERICAL EXAMPLE}

Considering a shallow shell in Fig. 1, which made of concrete with the geometry and material data: $\gamma=2500 \mathrm{~kg} / \mathrm{m}^{3} ; \mu=0.3 ; E=2.65 \times 10^{3}$ tit $\mathrm{kN} / \mathrm{cm}^{2} ; a=4 \mathrm{~m} ; b=6$ $\mathrm{m} ; R_{x}=R_{y}=12 \mathrm{~m} ; h=12 \mathrm{~cm} ; g=10 \mathrm{~m} / \mathrm{s}^{2}$. The shell is under axial forces $P_{x}=-1$ $\mathrm{kN} / \mathrm{cm}^{2}, P_{y}=-2 \mathrm{kN} / \mathrm{cm}^{2}$ uniformly distributed along the boundaries, respectively in $x$ and $y$ directions. The shell is also supposed to be excited by a force with amplitude $f_{0}=0.1 \mathrm{kN} / \mathrm{cm}^{2}$.

The diagnosis results for the axial forces $P_{x}, P_{y}$ and amplitude of external force $f_{0}$ are given in the columns $2-5$ of the below Table 1

Table 1. The deagnosis results of example

\begin{tabular}{|c|c|c|c|c|}
\hline \multirow{2}{*}{$\begin{array}{c}\text { Number of } \\
\text { frequencies }\end{array}$} & \multicolumn{5}{|c|}{ The diagnosis results for the axial forces $P_{x}, P_{y}$ and amplitude of } \\
measured & Error 0\% & Error $2 \%$ & Error $5 \%$ & Error $7 \%$ \\
\hline \multirow{3}{*}{3} & -1.000 & -1.159 & -1.215 & -1.359 \\
& -2.000 & -2.107 & -2.219 & -1.803 \\
& 0.100 & 0.095 & 0.087 & 0.080 \\
\hline \multirow{3}{*}{2} & -1.185 & -1.207 & -0.883 & -1.309 \\
& -1.907 & -1.956 & -1.831 & -2.698 \\
& 0.119 & 0.088 & 0.078 & 0.069 \\
\hline
\end{tabular}




\section{CONCLUSIONS}

From the above table, we see:

a. The method considering a combination between the direct and inverse problems of nonlinear vibration is effective one in the technical diagnosis problem of structure. The method is not only enough in determining the required parameters, but in agreement between the measured data and model parameters.

b. The analytical solution shows the relation between the parameters (known and unknown) of the technical diagnosis problem, that are loading, geometry, material and the parameters represented for vibration such as frequency, amplitude.

c. By using the nonlinear optimization methods for solving the technical diagnosis problem, we can obtain the accurate and stable results.

- The computed results show the high accuracy and stability of the solution. The introduction of the inequilibrium constraints lead to results with physical and mechanical meaning.

- The number of measured frequencies used in diagnosis are not necessary greater or equals to the number of parameters required to determine. However, the more number of frequencies is measured the more accurate diagnosis result is. It is also a remarkable feature of the dynamic testing methods.

d. The obtained results allow us to reevaluate the stress and strain state in plates and shells, from that we can evaluate the remaining capability of these structures.

\section{REFERENCES}

1. T. Colleman, M. A. Branch, A. Grace, Optimization Toolbox for Use with MATLAB. User's Guide Ver 2.0, The MATWORKS Inc., 1999.

2. S. Rao Singiresu, Mechanical Vibrations, Second Editions. Addison-Wesley, 1990.

3. A. C. Volmir, Neliniaia Dynamika Plactinok i Obolotrec, Mockva, 1972.

4. Nguyen Van Khang, Technical Vibration, Science and Technical Pub., 1998.

5. Le Ngoc Thach, Tran Van Lien, Chu Thanh Binh, The analysis of nonlinear forced vibration of plates and its in technical diagnosis, Building Science and Technology Journal 2 (2004) 32-35.

6. Nguyen Van Pho, Le Ngoc Hong, Le Ngoc Thach, On the numerical methods for solving technical diagnosis based on the inverse problems of mechanics, Proceedings of the $6^{\text {th }}$ National Conference on Mechanics, Hanoi, 391-408, 1997.

7. Le Ngoc Thach, On the method of technical diagnosis for the plate structures based on the direct and inverse problems of nonlinear vibrations, Proc. of the $7^{\text {th }}$ National Conference on Mechanics, Hanoi, 548-552, 2002. 


\section{DAO ĐỘNG PHI TUYẾN CỮ̛́NG BƯC CƯA VỎ THOẢI VÀ ỨNG DỤNG TRONG CHẦN DOÁN KỸ THUẬT}

Báo cáo trình bày bài toán thuận và ngược về dao động phi tuyến cưỡng bức của vơ thoải. Từ đó nêu cách thành lập và giải bài toán chẩn đoán kỹ thuật cho kết cấu vỏ thoải chịu tác dụng của tải trọng gây dao động. Tiêu chuẩn chẩn đoán là tổng bình phương sai lệch giữa kết qủa thực nghiệm và lý thuyết phải là bé nhất. Đại lượng được chẩn đoán là tải trọng tác dụng lên kết cấu và các phản lực phát sinh tại liên kết biên. 